\title{
Benzoxime inhibits matrix metalloproteinase-13 activation and cartilage damage in osteoarthritis rats via inhibition of NF-KB pathway
}

\author{
Tao He ${ }^{1}$, Zhi-ming $\mathrm{Li}^{1}$, Ming Li ${ }^{2 *}$, Ting-bin Yan ${ }^{3}$ \\ ${ }^{1}$ Department of Orthopedic Surgery, ${ }^{2}$ Department of Pathology, Dezhou People's Hospital, Dezhou, Shandong 253014. \\ ${ }^{3}$ Department of Orthopedic Surgery, Qilu Hospital, Shandong University, Jinan, Shandong 25000, China \\ *For correspondence: Email: mingli1821@hotmail.com
}

\begin{abstract}
Purpose: To investigate the effect of benzoxime on degradation of articular cartilage in a rat model of osteoarthritis (OA), and the mechanism involved.

Methods: The OA rat model was prepared by injecting monosodium iodoacetate (MIA) intra-articularly to Wistar rats. Rats in the treatment group were given benzoxime $(5 \mathrm{mg} / \mathrm{kg})$ daily for 21 days through the intra-articular route. The animals were then examined for behavioral changes by assessment of asymmetry in bearing weight and paw withdrawal threshold of the hind limb. Western blot assay was used for the analysis of inflammatory cytokine expressions.

Results: The expression of $P 2 X$ purinoceptor 7 receptor ( $P 2 X 7 R)$ mRNA was significantly elevated in the $O A$ rats $(p<0.02)$. However, benzoxime treatment caused a marked decrease in the level of P2X1$8 R$ mRNA. Benzoxime treatment also prevented asymmetry in bearing weight, decreased paw withdrawal threshold, and inhibited the expressions of interleukin-1 $\beta$, interleukin- 6 and tumor necrosis factor- $\alpha$ in plasma and cartilage. Moreover, benzoxime exhibited significant inhibitory effects on the expressions of P2X7R, matrix metalloproteinase (MMP)-13 and prostaglandin E2 (PGE2) in cartilage tissue. It also significantly suppressed OA-induced increases in the levels of inhibitor of nuclear factor$\kappa B(N F-K B)$ kinase $(I K K) \alpha$, IKKB, IKBa and NF-KB p65, and blocked OA-induced increases in the expressions of P2X7R, MMP-13 and PGE2.

Conclusion: These results demonstrate that benzoxime prevents cartilage degradation in $\mathrm{OA}$ rats by targeting NF-KB signaling pathway. Thus, benzoxime possesses clinical and therapeutic potentials for the prevention of cartilage degradation in $O A$.
\end{abstract}

Keywords: Interleukin-1ß, Purinoceptor-7, Benzoxime, Osteoarthritis, Prostaglandin, Matrix metalloproteinases

\begin{abstract}
This is an Open Access article that uses a funding model which does not charge readers or their institutions for access and distributed under the terms of the Creative Commons Attribution License (http://creativecommons.org/licenses/by/4.0) and the Budapest Open Access Initiative (http://www.budapestopenaccessinitiative.org/read), which permit unrestricted use, distribution, and reproduction in any medium, provided the original work is properly credited.

Tropical Journal of Pharmaceutical Research is indexed by Science Citation Index (SciSearch), Scopus, International Pharmaceutical Abstract, Chemical Abstracts, Embase, Index Copernicus, EBSCO, African Index Medicus, JournalSeek, Journal Citation Reports/Science Edition, Directory of Open Access Journals (DOAJ), African Journal Online, Bioline International, Open-J-Gate and Pharmacy Abstracts
\end{abstract}

\section{INTRODUCTION}

Osteoarthritis (OA), which is characterized by alteration in the architecture and composition of joints has adverse impact on socio-economic development $[1,2]$. The symptoms of early-stage $\mathrm{OA}$ include joint inflammation, edema, stiffness and pain, while the advanced stage is 
characterized by deformity of the joints $[1,2]$. The pathological alterations associated with $\mathrm{OA}$ are articular cartilage degradation, damage to subchondral bone and hyperplasia of the synovium [2]. Changes in the equilibrium between formation and degradation of cartilage matrix components are linked to etiology of bone tissue damage [3].

The current treatment for $O A$ involves conservative measures like modification of lifestyle, physiotherapy, chemotherapy and surgery [4]. At the early stage of $O A$, treatment involves symptomatic relief using available chemotherapeutic agents, while the advanced stage (characterized by irreversible disability of joints) requires surgery to relieve pain and improve joint function [5]. The development and progression of $\mathrm{OA}$ are induced by the expression of cytokines through alteration in the equilibrium between formation and degradation of chondrocytes [6]. Up-regulation of interleukin-13 leads to the breakdown of cartilage matrix and affects chondrocyte function [6,7]. The chondrocytes and synovial membrane of $\mathrm{OA}$ patients express higher level of interleukin- $1 \beta$, interleukin-6, tumor necrosis factor- $\alpha$ and prostaglandin E2 [8].

The expression of matrix metalloproteinases (which degrade cartilage tissues) is enhanced by prostaglandin E2 [9]. The currently-used nonsteroidal anti-inflammatory drugs for $O A$ treatment are associated with development of complications, while steroidal agents do not show satisfactory results [9]. Thus, the need for identification of newer and more effective chemotherapeutic agents for OA has continued to be a challenge to clinicians. Purinergic membrane receptor P2X expression in cartilage tissues has been shown to be associated with the onset of pain in OA patients [10]. The P2X7 receptor (P2X7R) plays an important role in the regulation of joint pain and edema [11]. It has been reported that damage to peripheral nerves causes up-regulation of P2X7R in macrophages [12].

The present study was undertaken to investigate the effect of benzoxime on cartilage tissue degradation in $\mathrm{OA}$ rats and the mechanism involved.

\section{EXPERIMENTAL}

\section{Animals}

Thirty, 8 - 10-week old male Wistar rats were obtained from the Shanghai SLAC Animal Co., Ltd., Shanghai. The rats were maintained singly in plastic boxes under $12 \mathrm{~h}$ light - 12h dark cycle at a constant temperature of $24{ }^{\circ} \mathrm{C}$ and humidity of $55 \pm 10 \%$, with ad libitum access to fresh water and standard laboratory feed. The rats were acclimatized to the laboratory conditions for one week before the start of the study. All animal experiments carried out according to the guidelines of Principles of international laboratory animal care [13]. The approval for the study was provided by the Research Ethical Committees of the Provincial Hospital Affiliated to Shandong University (approval no. TKY03331).

\section{Preparation of OA rat model and treatment}

Twenty rats exposed to volatile isoflurane anesthesia were administered $5 \mathrm{mg} / \mathrm{kg}$ MIA through the intra-articular route. The rats were then assigned to two groups of 10 animals each: benzoxime treatment group and model control group. The rats in the treatment group were intraarticularly injected $5 \mathrm{mg} / \mathrm{kg}$ benzoxime daily for 21 days. After analysis of behavioral changes for 21 days, the rats were sacrificed for western blot, enzyme-linked immunosorbent assay (ELISA) and quantitative reverse transcription polymerase chain reaction (RT-qRCR).

\section{RT-qPCR}

After treatment with benzoxime for 21 days, the $O A$ rats were sacrificed by spinal dislocation. The limb skin was disinfected to intercept the femur $5 \mathrm{~cm}$ from femoral condyle. Subsequently the tibia was also dissected below the tibia plateau to isolate the knee joint. The tissues around the joint were cleaned to expose the articular cartilage which was then carefully cut off from the joint. The RNA present in the cartilage was then isolated by TRIzol reagent (BD Biosciences) in accordance with the instructions on the kit manual. Samples of the RNA (5 $\mu \mathrm{g}$ samples) were subjected to reverse transcription using the PrimeScript RT reagent kit (Sigma-Aldrich, St. Louis, MO, USA). A mixture of 5X PrimeScript buffer $(2 \mu \mathrm{L})$, PrimeScript RT enzyme mix $(0.5$ $\mu \mathrm{L})$, total RNA $(2 \mu \mathrm{L})$ and RNase-free $\mathrm{dH}_{2} \mathrm{O}(5$ $\mu \mathrm{L})$ was incubated at room temperature for $20 \mathrm{~min}$, followed by heating to $85^{\circ} \mathrm{C}$ for $20 \mathrm{~s}$.

SYBR Premix Ex Taq TM II kit (RR041A; SigmaAldrich) was used for quantitative polymerase chain reaction. The cDNA $(2 \mu \mathrm{L})$ was combined with SYBR Premix Ex Taq $(12.5 \mu \mathrm{L}), 1 \mu \mathrm{l}$ each of forward and reverse primers, and $\mathrm{dH}_{2} \mathrm{O}(8.5 \mu \mathrm{L})$. The mixture was subjected to 40 cycles: initially 5 min at $95{ }^{\circ} \mathrm{C}$, then denaturation for $30 \mathrm{sec}$ at 95 ${ }^{\circ} \mathrm{C}$, annealing for $40 \mathrm{sec}$ at $60{ }^{\circ} \mathrm{C}$, and extension for $30 \mathrm{sec}$ at $72{ }^{\circ} \mathrm{C}$. After completion, 71 more cycles were performed at $80{ }^{\circ} \mathrm{C}$ for $30 \mathrm{sec}$. 
Subsequently, the temperature was increased by $0.5{ }^{\circ} \mathrm{C}$ for each repetition. Protein expression was normalized to GAPDH mRNA. The expressions of genes were quantified using SYBR Green Master mix (Life Technologies, Carlsbad, CA, USA).

\section{Behavioral assessments}

Following benzoxime treatment for 21 days, the OA rats were examined for asymmetry in bearing weight for the hind limb, and their paw withdrawal thresholds were evaluated. Asymmetry in bearing weight is expressed as the percentage of the distribution of weight between left and right hind limbs. The reference level was taken as the value obtained at the start of treatment. In the determination of paw withdrawal threshold, the animals were put on a steel wire mesh in an organic transparent glass box of dimensions 20 $\mathrm{cm} \times 20 \mathrm{~cm} \times 30 \mathrm{~cm}$. Von Frey monofilaments (58011, Stoelting Co., USA) were employed for the measurement of paw withdrawal threshold. For each animal, three readings were recorded for both hind limbs, and the mean value was calculated. The difference between the two hind limbs was used to determine paw withdrawal threshold.

\section{Analysis of knee edema size}

Edema sizes of the knee in the treatment, OA and vehicle control groups were determined after 21 days of treatment. Knee diameter was determined with a digital caliper and recorded for each rat. The diameter of the left knee was then subtracted from that of right knee to obtain the knee edema [14].

\section{Determination of cytokine expressions}

Blood samples were collected from the carotid artery of the animals under isoflurane anesthesia in EDTA phlebotomy blood sample bottles (Jiangsu Yuli Medical instruments Co., Ltd, China). The blood samples were centrifuged for $20 \mathrm{~min}$ at 3,500 $\times \mathrm{g}$ to obtain plasma samples which were stored under liquid nitrogen atmosphere. Cytokine levels in the plasma samples were determined using ELISA kits (Santa Cruz Biotechnology Inc., CA, USA) as per the manufacturer's protocol.

\section{Western blot analysis}

Sliced cartilage tissues from the treatment, OA and vehicle groups of rats were subjected to homogenization in TRIzol ${ }^{\circ}(50 \mathrm{mg}$ fresh weight/mL; Invitrogen, Paisley, UK) in liquid nitrogen using a dismembrator (Sartorious, Epsom, UK). The tissue homogenates were frozen, treated with lysis buffer and centrifuged for $10 \mathrm{~min}$ at $12,000 \times \mathrm{g}$ at $4{ }^{\circ} \mathrm{C}$ to remove the sediments. The protein contents of the supernatant fractions were determined with BCA protein assay kits. Thereafter, protein samples (25 - $35 \mu \mathrm{g}$ ) were subjected to electrophoresis on $12 \%$ sodium dodecyl sulfate-polyacrylamide gel, and subsequently transferred to polyvinylidene difluoride (PVDF) membranes. Membrane blocking was performed for $2 \mathrm{~h}$ at $36{ }^{\circ} \mathrm{C}$ using 3 $\%$ non-fat milk.

The membranes were incubated overnight with primary anti-bodies at a temperature of $4{ }^{\circ} \mathrm{C}$. The following primary antibodies were used: P2X7R (dilution 1:800; catalog no. P8232; SigmaAldrich), MMP-13 (dilution 1: 1, 000; catalog no. sc-2119; BD Biosciences), PGE2 (dilution 1: 1, 000; catalog no. ab176140; Abscam), IL-1 $\beta$ (dilution 1: 500; catalog no. KAC1211; Thermo Fischer Scientific), IL-6 (dilution 1: 500; catalog no. PHC0066; Thermo Fischer Scientific), TNF-a (dilution 1: 500; catalog no. KAC1751), IKKa (dilution 1: 600; catalog no. 14A231; Merck), pIKKa (dilution 1: 500; catalog no. 16A6; Cell Signaling Technology), IKK $\beta$ (dilution 1: 1, 000; catalog no. SC8330; Santa Cruz), p-IKK $\beta$ (dilution 1: 400; catalog no. AB55341; Abscam), IKBa (dilution 1: 800; catalog no. 2118S; Cell Signaling Technology), p-ІкBa (dilution 1:500; catalog no. 2859; Cell Signaling Technology), NF-kBp65 (dilution 1: 600; catalog no. KHO0371; Thermo Fischer Scientific), p-NF-kBp65 (dilution 1: 500; catalog no. 3033; Cell Signaling Technology) and GAPDH (dilution 1: 2, 000, BD Biosciences). The membranes were washed two times with PBS, and incubated for $2 \mathrm{~h}$ at ambient temperature with secondary antibodies conjugated with HRP. Visualization of the protein bands was achieved using an enhanced chemiluminescence detection system. Analysis of the densitometric values was done by the use of Image-ProPlus 6.0 (Media Cybernetics, Inc., Rockville, MD, USA). Normalization was done with GAPDH.

\section{Statistical analysis}

Data are presented as mean \pm standard deviation (SD) and were analyzed using one-way analysis of variance (ANOVA) and Bonferroni tests for comparison among more than two groups. Comparison between two groups was performed using Student's $t$-tests, with SPSS 13.0 (SPSS, Inc., Chicago, IL, USA). Differences were considered statistically significant at $p<$ 0.05 . 


\section{RESULTS}

\section{Effect of benzoxime on OA rats}

Rats in the control group showed no changes in asymmetry in bearing weight and paw withdrawal thresholds during the 21 days of study (Figures 1 $A$ and $B$ ). On the other hand, MIA administration significantly increased asymmetry in bearing weight to $32 \%$ on day 21 (Figure $1 \mathrm{~A} ; p<0.05$ ), and significantly decreased paw withdrawal threshold $(p<0.05)$ on day 21 , when compared to the control group (Figure 1B). The MIA administration also led to a significant increase in knee edema within the same period (Figure $1 \mathrm{C}$; $p<0.05)$. Administration of benzoxime to OA rats reversed the effects produced by MIA (Figure 1A). Benzoxime administration blocked the MIA-induced increases in weight bearing asymmetry and increased paw withdrawal threshold $(p<0.05)$. Moreover, benzoxime significantly reduced knee edema in the OA rats, relative to rats administered MIA alone $((p<$ 0.05, Figure 1C).

\section{Benzoxime prevented up-regulation of P2X7R expression in OA rats}

In the normal rats, the expression of P2X1-8R mRNA was slightly higher in cartilage tissues when compared to other members of P2X family (Figure 1 D). However, the expression of P2X18R mRNA was significantly higher in the OA rats, relative to the other members of this family $(p<$ 0.05 , Figure $1 \mathrm{D})$. Benzoxime treatment of the $O A$ rats caused significant decreases in the levels of P2X1-8R mRNA, when compared to the untreated control group.

\section{Benzoxime inhibited interleukin-1 $\beta$, interleukin-6 and tumor necrosis factor- $\alpha$ expressions in OA rat plasma}

Analysis of cytokine expression in OA rat plasma showed significantly higher levels of interleukin$1 \beta$, interleukin- 6 and tumor necrosis factor- $\alpha(p<$ 0.02). However, intra-articular administration of benzoxime to the OA rats prevented the MIAinduced increases in the expressions of interleukin- $1 \beta$, interleukin- 6 and tumor necrosis factor- $\alpha$ (Figure 2).

\section{Benzoxime inhibited expressions of cytokines in the cartilage tissues of OA rats}

Western blot analysis showed significantly higher expressions of interleukin-1 $\beta$, interleukin- 6 and tumor necrosis factor- $\alpha$ in cartilage tissues of $O A$ rats, when compared to the normal rats (Figure $3)$. The expressions of interleukin-1 $\beta$, interleukin-
6 and tumor necrosis factor- $\alpha$ in the cartilage tissues of OA rats were markedly down-regulated by administration of benzoxime $(p<0.01$, Figure 3).
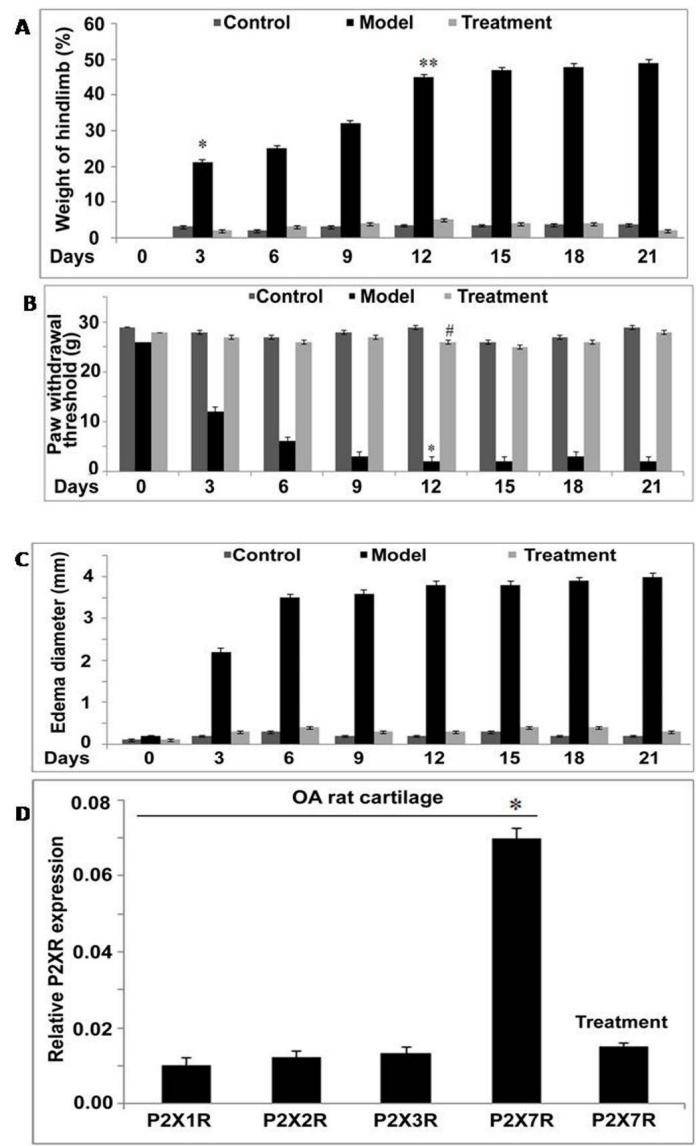

Figure 1: Effect of benzoxime on behavioral changes and expression of P2X1-8R mRNA in OA rats. (A) OA rats showed increase in asymmetry of bearing weight which was prevented by benzoxime administration. (B) In OA rats, benzoxime increased paw withdrawal thresholds and (C) inhibited edema formation. ${ }^{*} p<$ 0.02 , untreated vs. normal; ${ }^{\#} p<0.05$, treatment group vs. untreated. (D) Benzoxime treatment for 21 days inhibited P2X1-8R mRNA expression in OA rats

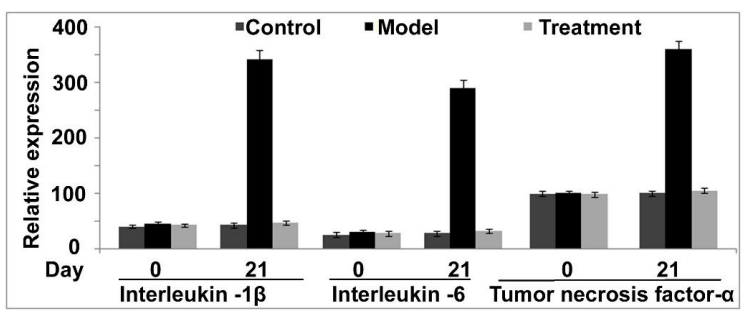

Figure 2: Effect of benzoxime on cytokine expression in OA rats. After 21 days of treatment, the levels of interleukin-1 $\beta$, interleukin- 6 and tumor necrosis factor$\alpha$ in the rat plasma were examined by ELISA. Benzoxime treatment inhibited the OA-induced upregulation of cytokines in the plasma. ${ }^{*} p<0.02$, untreated vs. normal; ${ }^{\#} p<0.02$, treatment vs. untreated 


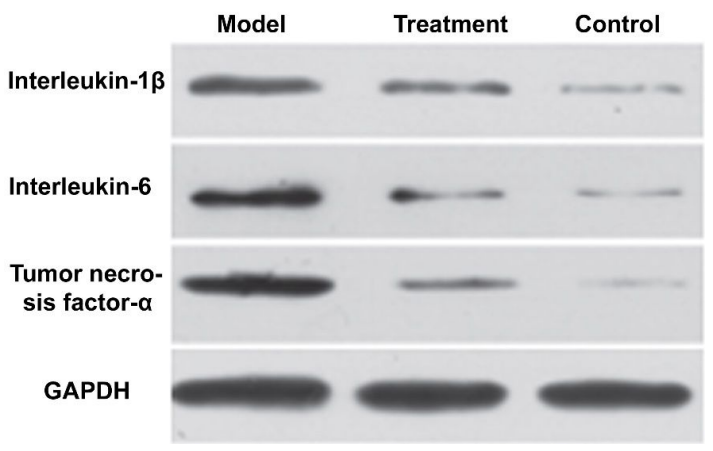

Figure 3: Effect of benzoxime on the expressions of pro-inflammatory factors in the cartilage tissues of $O A$ rats. The cartilage tissues of OA rats contained higher levels of interleukin-1 $\beta$, interleukin- 6 and tumor necrosis factor- $\alpha$. Treatment of $O A$ rats with benzoxime for 21 days inhibited OA-mediated upregulation of these cytokines

\section{Benzoxime inhibited P2X7R, matrix metalloproteinase-13 and PGE2 expressions in OA rats}

The expressions of P2X7R, matrix metalloproteinase-13 and PGE2 in the cartilage tissue were significantly higher in OA rats than in normal control rats $(p<0.05$, Figure 4). However, benzoxime treatment exerted inhibitory effects on the expressions of these proteins (P2X7R, matrix metalloproteinase-13 and PGE2) in the cartilage tissues of OA rats (Figure 4). Significant decreases in the expression of P2X7R, MMP-13 and PGE2 were also observed in $\mathrm{OA}$ rats administered helenalin, a nuclear factor-kB (NF-kB) inhibitor.

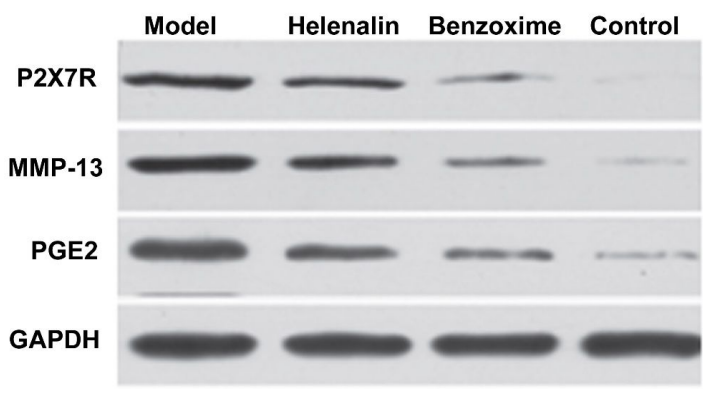

Figure 4: Effect of benzoxime treatment on the expressions of PGE2, MMP-13 and P2X7R expression in cartilage tissues of $\mathrm{OA}$ rats

\section{Effect of benzoxime on the expressions of

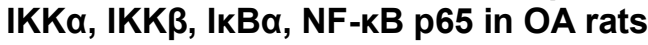

Results from western blot assay showed significant increases in the expressions of IKKa, IKK $\beta$, IKB $\alpha$, and NF-KB $\mathrm{p} 65$ in the cartilage tissues of $\mathrm{OA}$ rats (Figure $5 ; p<0.05$ ). The expressions of activated IKKa, IKK $\beta$, IKB $\alpha$ and NF-KB p65 were significantly increased in the OA rats, when compared to normal rats. However, benzoxime administration to $\mathrm{OA}$ rats suppressed the increases in the levels of IKKa, IKK 3 , IKBa and NF-KB p65 in the OA rats (Figure 5).

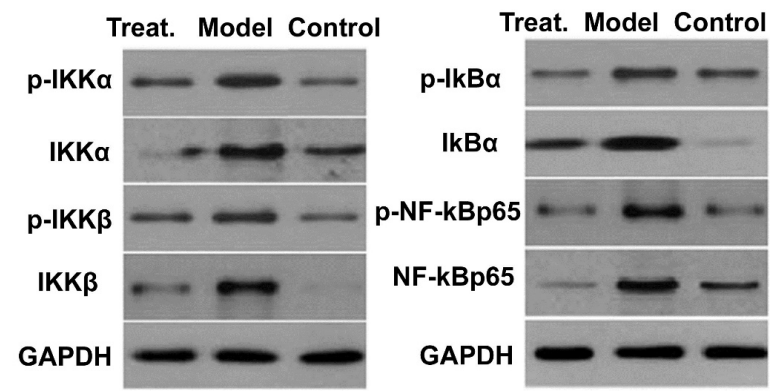

Figure 5: Effect of benzoxime on the expressions and phosphorylation of IKKa, IKBa NF-KBp65 and IKK $\beta$ in $\mathrm{OA}$ rats. The expressions and activation of these molecules were significantly enhanced in OA rats, but were inhibited by benzoxime treatment

\section{DISCUSSION}

It has been reported that inhibition of the expression of inflammatory cytokines prevents the decomposition of cartilage tissues [15]. The characteristic features of $O A$ include knee joint edema, decomposition of cartilage, hyperplasia of synovium and degradation of sub-chondral bones [16]. Various cytokines such as nitric oxide, PG, interleukin-1 $\beta$, tumor necrosis factor$\alpha$, interleukin- 6 and interleukin-8 are expressed in higher proportions in the chondrocytes of $O A$ patients $[6,9]$. Chondrocytes play an important role in the onset of pain during inflammation by activating the neurons $[7,16]$. Studies have demonstrated that the expressions of interleukin4, tumor necrosis factor- $\alpha$, interleukin- 6 , and interleukin-13 are promoted by P2X7R activation [17].

Down-regulation of tumor necrosis- $\alpha$ also leads to longer inhibition of inflammation [18]. It has been observed that P2X7R is linked to inflammation of knee joints and development of OA [10]. The current study has demonstrated that pain associated with $\mathrm{OA}$ in rats can be successfully suppressed by treatment with benzoxime. In addition, edema formation in the OA was prevented by treatment with benzoxime through inhibition of inflammatory processes. It was also demonstrated that in $\mathrm{OA}$ rats the expression of P2X1-8R mRNA was significantly higher and that of other members of P2X receptors.

Benzoxime administration blocked MIA-induced increases in asymmetry in bearing weight and decreased paw withdrawal threshold. In addition, benzoxime administration to $\mathrm{OA}$ rats significantly prevented knee edema, when compared to rats 
administered MIA alone. In OA patients benzoxime exhibits inhibitory effect on inflammation, and PGE2 upregulates degradation of cartilage by promoting the expressions of matrix metalloproteinases [9]. The level of matrix metalloproteinase-13 expression in $\mathrm{OA}$ patients is considered to be a reliable indicator of the extent of degradation of cartilage [19].

The expression of metalloproteinase- 13 was significantly higher in the OA but was inhibited by treatment with benzoxime. It has been reported that the expression of interleukin-1 $\beta$ in the chondrocytes causes activation of NF-KB pathway [20]. Studies have also revealed that the NF-KB signaling pathway is activated by expression of P2X7R in OA patients [21]. In the present study, the activation of IKKa, IKK $\beta$, IKBa, NF-KB p65 was promoted in OA rats. However, treatment of the $\mathrm{OA}$ rats with benzoxime prevented up-regulation of these molecules. The inactive form of NF-KB exists in the cytoplasm and on activation by P2X7R the molecule enters the nucleus [22]. This study has demonstrated that expression of P2X7R, matrix metalloproteinase-13 and PGE2 in the cartilage tissues of $O A$ rats were significantly higher than their corresponding levels in normal rats.

Administration of benzoxime exhibited inhibitory effects on the expression of P2X7R, matrix metalloproteinase-13 and PGE2 in the cartilage tissues of $O A$ rats. Western blot assay showed that the expression of IKKa, IKK $\beta$, IKBa, NF-KB p65 were increased in the cartilage tissues of $O A$ rats. The expressions of activated IKKa, IKK $\beta$, IKB $\alpha$ and NF-KB p65 were increased significantly in the $O A$ rats, relative to normal rats. However, benzoxime administration suppressed the increased levels of IKKa, IKK $\beta$, IKB $\alpha$ and NF-KB p65 in the OA rats.

\section{CONCLUSION}

The findings of the current study demonstrate that benzoxime prevents cartilage degradation in $\mathrm{OA}$ rats through targeting NF-KB signaling pathway. This implies that benzoxime possesses clinical and therapeutic potential for the management of cartilage degradation associated with $\mathrm{OA}$.

\section{DECLARATIONS}

\section{Acknowledgement}

The authors are thankful to their Heads of Departments for support.

\section{Conflict of interest}

The authors declare that no conflict of interest is associated with this study.

\section{Author contributions}

We declare that this work was done by the authors named in this article and all liabilities pertaining to claims relating to the content of this article will be borne by the authors. Tao $\mathrm{He}$ and Zhi-ming Li performed the experimental work and Ming $\mathrm{Li}$ designed the study. Ting-bin Yan compiled the results and did literature study. Ming Li and Ting-bin Yan wrote the paper. All the authors read and approved the manuscript before its submission for publication.

\section{REFERENCES}

1. Gabriel SE, Crowson CS, Campion ME, O'Fallon WM. Direct medical costs unique to people with arthritis. J Rheumatol 1997; 24: 719-725.

2. March LM, Bachmeier CJ. Economics of osteoarthritis: a global perspective. Baillieres Clin Rheumatol 1997; 11: 817-834.

3. Martel-Pelletier J, Lajeunesse $d$, Pelletier JP. Etiopathogenesis of osteoarthritis. In: Arthritis and Allied conditions: A Textbook of Rheumatology. Vol 2. 15th edition. Koopman WJ and Moreland LW (eds). Lippincott Williams \& Wilkins, Philadelphia, 2005; 21992226.

4. Puhl W, Bernau A, Greiling H, Köpcke W, Pförringer W, Steck KJ, Zacher J, Scharf HP. Intra-articular sodium hyaluronate in osteoarthritis of the knee: a multicenter, double-blind study. Osteoarthritis cartilage 19931; 233241.

5. Buckwalter JA, Mankin HJ. Articular cartilage: degeneration and osteoarthritis, repair, regeneration, and transplantation. Instr course Lect 1998; 47: 487504.

6. Goldring MB. Osteoarthritis and cartilage: the role of cytokines. Curr Rheumatol Rep 2000; 2: 459-465.

7. Largo R, Alvarez-Soria MA, Díez-Ortego I, Calvo E, SánchezPernaute $O$, Egido J, Herrero-Beaumont $G$. Glucosamine inhibits IL-1beta-induced NF-kappaB activation in human osteoarthritic chondrocytes. Osteoarthritis Cartilage 2003; 11: 290-298.

8. Pelletier JP, Martel-Pelletier J, Abramson SB. Osteoarthritis, an inflammatory disease: potential implication for the selection of new therapeutic targets. Arthritis Rheum 2001; 44: 1237-1247.

9. Bar-Or D, Rael LT, Thomas GW, Brody EN. Inflammatory pathways in knee osteoarthritis: potential targets for treatment. Curr Rheumatol Rev: 2015.

10. Staunton CA, Lewis R, Barrett-Jolley R. Ion channels and osteoarthritic pain: potential for novel analgesics. Curr Pain Headache Rep 2013; 17: 378. 
11. Bravo D, Maturana CJ, Pelissier T, Hernández A, Constandil L. Interactions of pannexin 1 with NMDA and $P 2 X 7$ receptors in central nervous system pathologies: possible role on chronic pain. Pharmacol Res 2015; 101: 86-93.

12. Dray A, Read SJ. Arthritis and pain. Future targets to control osteoarthritis pain. Arthritis Res Ther 2007; 9: 212.

13. Guide for the Care and Use of Laboratory Animals: Eighth Edition Committee for the Update of the Guide for the Care and Use of Laboratory Animals; National Research Council. 2010; ISBN: 0-309-15401-4.

14. Fernihough J, Gentry C, Malcangio M, Fox A, Rediske J, Pellas T, Kidd B, Bevan S, Winter J. Pain related behaviour in two models of osteoarthritis in the rat knee. Pain 2004; 112: 83-93.

15. Keystone EC, Wang MM, Layton M, Hollis S, Mclnnes IB. D1520C00001 Study Team: Clinical evaluation of the efficacy of the $P 2 X 7$ purinergic receptor antagonist AZD9056 on the signs and symptoms of rheumatoid arthritis in patients with active disease despite treatment with methotrexate or sulphasalazine. Ann Rheum Dis 2012; 71: 1630-1635.

16. Konttinen $Y T$, Sillat $T$, Barreto $G$, Ainola $M$, Nordström $D C$. Osteoarthritis as an autoinflammatory disease caused by chondrocyte-mediated inflammatory responses. Arthritis Rheum 2012; 64: 613-616.
17. Lister MF, Sharkey J, Sawatzky DA, Hodgkiss JP, Davidson DJ, Rossi AG, Finlayson K. The role of the purinergic $P 2 X 7$ receptor in inflammation. J Inflamm (Lond) 2007; 4: 5.

18. Catal F, Mete E, Tayman C, Topal E, Albayrak A, Sert H. A human monoclonal anti-TNF alpha antibody (adalimumab) reduces airway inflammation and ameliorates lung histology in a murine model of acute asthma. Allergol Immunopathol (Madr) 2015; 43: 14-18.

19. Malemud CJ, Islam N, Haqqi TM. Pathophysiological mechanisms in osteoarthritis lead to novel therapeutic strategies. Cells Tissues Organs 2003; 174: 34-48.

20. Csaki C, Mobasheri A, Shakibaei M. Synergistic chondroprotective effects of curcumin and resveratrol in human articular chondrocytes: inhibition of IL-1betainduced NF-kappaB-mediated inflammation and apoptosis. Arthritis Res Ther 2009; 11: R165.

21. He $C$, Chen $X$, Zhao $C$, Qie $Y$, Yan Z, Zhu $X$. Eleutheroside $E$ ameliorates arthritis severity in collagen-induced arthritis mice model by suppressing inflammatory cytokine release. Inflammation 2014; 37: 1533-1543.

22. Ferrari D, Wesselborg S, Bauer MK, Schulze-Osthoff $K$. Extracellular ATP activates transcription factor NFkappaB through the $P 2 Z$ purinoreceptor by selectively targeting NF-kappaB p65. J Cell Biol 1997; 139: 16351643. 